\title{
MOTION PLANNING IN CARTESIAN PRODUCT GRAPHS
}

\author{
Biswajit DeB ${ }^{1}$ \\ Department of Mathematics \\ Sikkim Manipal Institute of Technology, India \\ e-mail: bdeb@iitg.ernet.in \\ AND \\ KALPESH KAPOOR \\ Department of Mathematics \\ Indian Institute of Technology Guwahati, India \\ e-mail: kalpesh@iitg.ernet.in
}

\begin{abstract}
Let $G$ be an undirected graph with $n$ vertices. Assume that a robot is placed on a vertex and $n-2$ obstacles are placed on the other vertices. A vertex on which neither a robot nor an obstacle is placed is said to have a hole. Consider a single player game in which a robot or obstacle can be moved to adjacent vertex if it has a hole. The objective is to take the robot to a fixed destination vertex using minimum number of moves. In general, it is not necessary that the robot will take a shortest path between the source and destination vertices in graph $G$. In this article we show that the path traced by the robot coincides with a shortest path in case of Cartesian product graphs. We give the minimum number of moves required for the motion planning problem in Cartesian product of two graphs having girth 6 or more. A result that we prove in the context of Cartesian product of $P_{n}$ with itself has been used earlier to develop an approximation algorithm for $\left(n^{2}-1\right)$-puzzle.
\end{abstract}

Keywords: robot motion in a graph, Cartesian product of graphs.

2010 Mathematics Subject Classification: 05C85, 05C75, 68R10, 91A43.

\footnotetext{
${ }^{1}$ This research was done while the author was a Ph.D. student at the Department of Mathematics, Indian Institute of Technology Guwahati, India. The author thanks Sikkim Manipal Institute of Technology and the All India Council for Technical Education (AICTE) to give paid-leave and provide financial support, respectively, for this study.
} 


\section{REFERENCES}

[1] G. Calinescu, A. Dumitrescu and J. Pach, Reconfigurations in graphs and grids, in: Latin American Theoretical Informatics Conference Lecture Notes in Computer Science 3887 (2006) 262-273.

doi:10.1007/11682462_27

[2] B. Deb, K. Kapoor and S. Pati, On mrj reachability in trees Discrete Math., Alg. and Appl. 4 (2012). doi:10.1142/S1793830912500553

[3] T. Feder, Stable Networks and Product Graphs (Memoirs of the American Mathematical Society, 1995). doi: $10.1090 / \mathrm{memo} / 0555$

[4] R. Hammack, W. Imrich and S. Klažar, Handbook of Product Graphs, Second Edition (CRC Press, New York, 2011).

[5] F. Harary, Graph Theory (Addison-Wesley, Reading, MA, 1969).

[6] W. Imrich and S. Klavžar, Product Graphs, Structure and Recognition (John Wiley \& Sons Inc., New York, 2000).

[7] W. Woolsey Johnson and W.E. Story, Notes on the "15" puzzle, Amer. J. Math. 2 (1879) 397-404. doi: $10.2307 / 2369492$

[8] D. Kornhauser, G. Miller, and P. Spirakis, Coordinating pebble motion on graphs, the diameter of permutation groups, and applications, in: Annual Symposium on Foundations of Computer Science, IEEE Computer Society (1984) 241-250. doi:10.1109/SFCS.1984.715921

[9] E. Masehian and A.H. Nejad, Solvability of multi robot motion planning problems on trees, in: IEEE/RSJ International Conference on Intelligent Robots and Systems, Piscataway, NJ, USA (2009), IEEE Press. 5936-5941. doi:10.1109/IROS.2009.5354148

[10] C.H. Papadimitriou, P. Raghavan, M. Sudan and H. Tamaki, Motion planning on a graph, in: FOCS'94 (1994) 511-520. doi:10.1109/SFCS.1994.365740

[11] I. Parberry, A real-time algorithm for the $\left(n^{2}-1\right)$-puzzle, Inform. Process. Lett. 56 (1995) 23-28. doi:10.1016/0020-0190(95)00134-X

[12] D. Ratner and M.K. Warmuth, The $\left(n^{2}-1\right)$-puzzle and related relocation problems, J. Symbolic Comput. 10 (1990) 111-137. doi:10.1016/S0747-7171(08)80001-6

[13] D. Ratner and M.K. Warmuth, Finding a shortest solution for the $n \times n$ extension of the 15-puzzle is intractable, in: AAAI(1986) 168-172.

[14] R.M. Wilson, Graph puzzles, homotopy, and the alternating group, J. Combin. Theory (B) 16 (1974) 86-96. doi:10.1016/0095-8956(74)90098-7 
Received 10 April 2012

Revised 5 February 2013

Accepted 5 February 2013 